\title{
New probes for axionlike particles at hadron colliders
}

\author{
Javad Ebadi, ${ }^{1, *}$ Sara Khatibi, ${ }^{2,3, \dagger}$ and Mojtaba Mohammadi Najafabadi ${ }^{3, \hbar}$ \\ ${ }^{1}$ School of Physics, Institute for Research in Fundamental Sciences, P.O. Box 19395-5531, Tehran, Iran \\ ${ }^{2}$ Department of Physics, University of Tehran, North Karegar Ave., Tehran 14395-547, Iran \\ ${ }^{3}$ School of Particles and Accelerators, Institute for Research in Fundamental Sciences (IPM) \\ P.O. Box 19395-5531, Tehran, Iran
}

(Received 17 March 2019; published 15 July 2019)

\begin{abstract}
Axionlike particles (ALPs) appear from spontaneous global symmetry breaking in many extensions of the Standard Model (SM). In this paper, we find bounds on ALP $(a)$ model parameters at the Large Hadron Collider (LHC) from the ALP production associated with a photon and a jet $(j+\gamma+a)$ as well as single top and top quark pairs $(t+j+a, t \bar{t}+a)$ in a model-independent approach. In particular, it is shown that the ALP production associated with a photon plus a jet at the LHC is a promising channel with significant sensitivity to probe the ALP couplings to gluons and electroweak gauge bosons. The prospects are presented at the high luminosity LHC including a realistic detector simulation and pileup effects. Furthermore, the ALP model is examined through its contributions to the top quark (chromo)magnetic dipole moments. It is shown that the top quark magnetic and chromomagnetic dipole moments enable us to probe the ALP couplings to top quark and gauge bosons at a time. The constraints are complementary to those obtained from direct searches, as they are sensitive to light ALPs.
\end{abstract}

DOI: 10.1103/PhysRevD.100.015016

\section{INTRODUCTION}

In spite of the remarkable achievements of the Standard Model (SM) of particles physics, there are observational and theoretical aspects which are not completely understood or explained within the SM framework. Existence of dark matter (DM), neutrino mass, and baryon asymmetry are examples of observational problems. The strong $C P$ and hierarchy problems are among the theoretical problems not addressed in the SM. So far, many theories beyond the Standard Model (BSM) have been constructed to explain these defects. Although numerous attempts have already been done to figure out a footprint of these BSMs at the LHC, no significant sign of new physics at high energies has been discovered yet. As a result, looking for new light degrees of freedom or weakly coupled states to the SM content are taken into consideration these days.

Many new models predict one or more new light pseudoscalar particles in their spectrum which enable us to explain part of the SM shortcomings. For instance, in order to solve the strong $C P$ problem [1-3], Peccei and

\footnotetext{
*javadebadi@ipm.ir sara.khatibi@ut.ac.ir ¥mojtaba@ipm.ir
}

Published by the American Physical Society under the terms of the Creative Commons Attribution 4.0 International license. Further distribution of this work must maintain attribution to the author(s) and the published article's title, journal citation, and DOI. Funded by SCOAP.
Quinn [4] proposed a mechanism with a spontaneously broken global $U(1)_{\mathrm{PQ}}$ symmetry, which predicts a pseudoNambu-Goldstone boson, called quantum chromodynamics axion. Furthermore, the pseudoscalar particles can appear in other models, such as DM portal models [5], low-energy effective field theories of string theory [6], and some models which explain the muon magnetic moment anomaly [7]. In general, any model with global $U(1)$ symmetries, which are spontaneously broken, predicts pseudo-Nambu-Goldstone bosons so that their masses and couplings are independent parameters. The pseudo-Nambu-Goldstone bosons in such models are called axionlike particles (ALPs). The strength of the couplings of the ALPs to SM matter fields is proportional to the inverse of the scale of $U(1)$ spontaneous symmetry breaking $f_{a}$, which is much larger than the SM electroweak symmetry breaking scale. The ALP model is able to solve the observed matter-antimatter asymmetry for the case that $f_{a}$ resides between about 100 and $10^{4} \mathrm{TeV}$ [8]. PseudoNambu-Goldstone bosons could also appear in supersymmetric (SUSY) models with SUSY dynamical breaking and a spontaneously broken R symmetry. Such pseudo-NambuGoldstone bosons are called $\mathrm{R}$ axion and naturally expected to be light $[9,10]$.

A considerable region of the parameter space of the ALP model has already been probed by cosmological observations and low-energy experiments. Also, the large electronpositron collider and LHC data have been employed to constrain the parameter space of ALP. If an ALP is produced in a collision at high-energy colliders, it has 
different properties which can be classified into four categories. First, ALP can be long-lived compared to the detector scales; therefore, it can escape detection which results in a large missing energy signature in the detectors. We note that ALPs have a negligible interaction with detector material due to very stringent bounds on their interactions with fermions [11]. Second, ALP can decay to two massless gauge bosons inside the detector, and the detector could discriminate between these two objects. The signature would contain two jets or two photons which are nearly back to back. This signature is expected if the ALP is heavy, and the extreme limit of this case could happen when the ALP is produced nearly at rest. Third, ALP can be highly boosted and decays to two massless gauge bosons inside the detector, but is recognized as one object in the detector. In the case of decay of ALP into two gluons, the signature would be a "fat jet." For the photons, there is a possibility that detector recognizes two photons as a single photon. Fourth, the ALP decays into a $Z$ boson and a photon or into two massive gauge bosons $V V(V V=Z Z, W W)$. In $a \rightarrow Z \gamma$, the partial decay width for light ALPs is suppressed by the $\left(m_{a} / m_{Z}\right)^{4}$ factor. More suppression for the case of $a \rightarrow V V$ is expected due to more limited phase space.

Decays $Z \rightarrow \gamma \gamma$ and $Z \rightarrow \gamma \gamma \gamma$ provide the possibility to exclude part of the parameter space of the ALP model which was not excluded by low-energy experiments $[12,13]$. Higgs exotic decay modes enable us to access part of the parameter space which could not be constrained by $Z$ boson decays. The $h \rightarrow Z a \rightarrow \ell^{+} \ell^{-} \gamma \gamma$ and $h \rightarrow a a \rightarrow \gamma \gamma \gamma \gamma$ channels at LHC are utilized to probe the ALP coupling to the photon in terms of the ALP mass $[14,15]$. Moreover, there are studies based on large missing energy signature where the ALP escapes detections and appears as missing momentum. The monojet, mono- $\gamma$, dijet, and diphoton searches at the LHC and future colliders are employed to constrain the ALP parameter space $[13,16]$. In addition, the mono- $W$, mono- $Z$, mono-Higgs channels, and the associated production of ALP with $W+\gamma$ are also investigated in Ref. [11]. There are other proposed ways to look for ALP at the colliders. For example, the heavy ion ultraperipheral collisions are utilized to search for axion coupling to photons [17-19]. Moreover, the ALP model parameter space could be probed in forward physics [20]. Flavor factories using $B$ meson decays and flavor changing processes are also power tools to probe the ALP couplings [21,22]. The ALP coupling with gluon has been studied based on a data-driven method for a region of ALP mass close to quantum chromodynamics scale to $3 \mathrm{GeV}$ in Ref. [23].

In this work, we study the associated production of an ALP with a photon and a jet, single top quark plus an ALP, and $t \bar{t}$ production with an ALP at the LHC to probe the ALP model parameter space. This study concentrates on a region of the ALP parameter space, in which it would not decay inside the detector and appear as missing transverse energy
$\left(E_{T}^{\text {miss }}\right)$. We also use the top quark (chromo)magnetic dipole moment as a tool to limit the ALP couplings.

The organization of the paper is as follows. In Sec. II, a short introduction of the theoretical framework of the ALP model is given. Section III presents the colliders searches for the ALP model using the associated production of an ALP with a jet and a photon as well as top quark(s). In Sec. IV, the contributions of the ALP model to the top quark magnetic and chromomagnetic dipole moments are calculated. Then, the upper limits on the (chromo)magnetic dipole moments are used to constrain the model parameters. Finally, a summary of the results and conclusions are given in Sec. V.

\section{THEORETICAL FRAMEWORK}

In this work, an ALP is studied in a scenario where it is a singlet scalar under the SM gauge group and is odd under the $C P$ transformation. The most general Lagrangian up to dimension $D=5$ operators which describe the effective interactions of the ALP and SM fields is given by [11]

$$
\begin{aligned}
\mathcal{L}_{\mathrm{eff}}^{D \leq 5}= & \mathcal{L}_{\mathrm{SM}}+\frac{1}{2}\left(\partial^{\mu} a\right)\left(\partial_{\mu} a\right)-\frac{1}{2} m_{a}^{2} a^{2} \\
& +c_{a \Phi} \frac{\partial^{\mu} a}{f_{a}}\left(\Phi^{\dagger} i \stackrel{\leftrightarrow}{D_{\mu}} \Phi\right)+\frac{\partial^{\mu} a}{f_{a}} \sum_{F} \bar{\Psi}_{F} \mathbf{C}_{F} \Psi_{F} \\
& -c_{G G} \frac{a}{f_{a}} G_{\mu \nu}^{A} \tilde{G}^{\mu \nu, a}-c_{B B} \frac{a}{f_{a}} B_{\mu \nu} \tilde{B}^{\mu \nu} \\
& -c_{W W} \frac{a}{f_{a}} W_{\mu \nu}^{a} \tilde{W}^{\mu \nu, a} .
\end{aligned}
$$

Here the summation is performed over all the SM fermions field $F=L_{L}, Q_{L}, e_{R}, d_{R}, u_{R}$, where $L_{L}$ and $Q_{L}$ are the $S U(2)_{L}$ doublets and $e_{R}, d_{R}, u_{R}$ are $S U(2)_{L}$ singlet. The $\mathbf{C}_{F}$ matrices are $3 \times 3$ Hermitian matrices in flavor space. The Higgs boson doublet is denoted by $\Phi$, and ALP field is represented by $a$. The $G_{\mu \nu}, W_{\mu \nu}$, and $B_{\mu \nu}$ are field strengths for $S U(3)_{c}, S U(2)_{L}$, and $U(1)_{Y}$, respectively. The $\tilde{X}^{\mu \nu}$ is defined as follows:

$$
\tilde{X}^{\mu \nu} \equiv \frac{1}{2} \epsilon^{\mu \nu \alpha \beta} X_{\alpha \beta},
$$

where $\epsilon^{\alpha \beta \mu \nu}$ is the Levi-Civita symbol. We note that the Lagrangian of Eq. (1) does not add any new source of $C P$ violation other than what already exists in the SM. This Lagrangian respects gauge symmetry of SM, and it is invariant under the $C P$ transformation. In addition, neglecting the ALP mass term, the Lagrangian is invariant under shift transformation up to total derivative terms which are a manifestation of a broken global symmetry. In this work, for simplicity we only focus on bosonic Lagrangian, or in other words, $\mathbf{C}_{F}=0$ are assumed for all five types of SM fermions. Then, after performing the following field redefinition: 


$$
\Phi \rightarrow e^{i c_{a \Phi} a / f_{a}} \Phi
$$

the $\frac{\partial^{\mu} a}{f_{a}}\left(\Phi^{\dagger} i \stackrel{\leftrightarrow}{D_{\mu}} \Phi\right)$ operator is eliminated which causes the appearance shift-symmetry breaking terms in the Lagrangian. After the field redefinition Eq. (2), the Lagrangian up to dimension five operators takes the following form [11]:

$$
\begin{aligned}
\mathcal{L}_{\text {eff }}^{D \leq 5}= & \mathcal{L}_{\mathrm{SM}}+\frac{1}{2}\left(\partial^{\mu} a\right)\left(\partial_{\mu} a\right)-\frac{1}{2} m_{a}^{2} a^{2} \\
& +-c_{G G} \frac{a}{f_{a}} G_{\mu \nu}^{A} \tilde{G}^{\mu \nu, A}-c_{W W} \frac{a}{f_{a}} W_{\mu \nu}^{A} \tilde{W}^{\mu \nu, A} \\
& -c_{B B} \frac{a}{f_{a}} B_{\mu \nu} \tilde{B}^{\mu \nu}+c_{a \Phi} \mathbf{O}_{a \Phi}^{\psi}
\end{aligned}
$$

where

$$
\mathbf{O}_{a \Phi}^{\psi} \equiv i\left(\bar{Q}_{L} \mathbf{Y}_{U} \tilde{\Phi} u_{R}-\bar{Q}_{L} \mathbf{Y}_{D} \Phi d_{R}-\bar{L}_{L} \mathbf{Y}_{E} \Phi e_{R}\right) \frac{a}{f_{a}}+\text { H.c. }
$$

The higher order terms, which appear due to the field redefinition, have been omitted in the Lagrangian.

The Lagrangian of Eq. (3) has been implemented in FeynRules [24] based on the study and notation of Ref. [11]. Then, the obtained universal FeynRules output $[25]^{1}$ model is inserted to MadGraph5_aMC@NLO [26] to perform the numerical calculations of the cross sections and to generate events.

\section{A. ALP decay}

ALP can decay into charged leptons, photons, jets (gluons, quarks), or light hadrons according to its mass and couplings to these particles. The Lagrangian describing the decay of ALP to two photons and two gluons can be written as follows:

$$
\mathcal{L}_{\text {eff }}^{D \leq 5} \supset-c_{\gamma \gamma} \frac{a}{f_{a}} F_{\mu \nu} \tilde{F}^{\mu \nu}-c_{G G} \frac{a}{f_{a}} G_{\mu \nu}^{A} \tilde{G}^{\mu \nu, A},
$$

where $F_{\mu \nu}$ and $G_{\mu \nu}^{A}$ are the field strengths of the photon and gluon, respectively. $c_{G G}$ and $c_{\gamma \gamma}$ are the couplings of gluon and photon with the ALP. The coupling with photon $c_{\gamma \gamma}$ is related to $c_{B B}$ and $c_{W W}$ of Eq. (3) via

$$
c_{\gamma \gamma}=\frac{1}{f_{a}}\left(c_{B B} \cos \theta_{W}^{2}+c_{W W} \sin \theta_{W}^{2}\right),
$$

where $\theta_{W}$ is the Weinberg angle. There are several studies to constrain the ALP mass and its coupling to photons $c_{\gamma \gamma}$ obtained from various experiments ranging from

\footnotetext{
${ }^{1}$ http://feynrules.irmp.ucl.ac.be/attachment/wiki/ALPsEFT/ ALP_linear_UFO.tar.gz.
}

low-energy experiments to high-energy colliders and cosmological observations [14,15].

The decay rates of an ALP into two photons and two gluons at leading order can be written as

$$
\Gamma_{a \rightarrow \gamma \gamma}=\left(\frac{c_{\gamma \gamma}}{f_{a}}\right)^{2} \frac{m_{a}^{3}}{4 \pi}, \quad \Gamma_{a \rightarrow g g}=8 \times\left(\frac{c_{G G}}{f_{a}}\right)^{2} \frac{m_{a}^{3}}{4 \pi},
$$

where the decay rate of $a \rightarrow g g$ is calculated using pQCD which only is valid providing that $m_{a} \gg \Lambda_{\mathrm{QCD}}$. Chiral perturbation theory can be used for studying the interaction of gluon with ALP for the case of $m_{a} \lesssim 1 \mathrm{GeV}$. In Ref. [23], a data-driven method has been proposed to determine $c_{G G}$ for the region of $m_{a}$ close to quantum chromodynamics scale to $3 \mathrm{GeV}$ using inclusive decays of $b \rightarrow s+a$, and $\phi, \eta^{\prime}, B^{ \pm}$, and $B^{0}$ decays. In this work, the ALP decay rate is obtained from Ref. [23] in which the calculations are based on chiral perturbation theory.

\section{ASSOCIATED PRODUCTION OF AN ALP AT THE LHC}

In this section, we concentrate on constraining the ALP model parameters through various processes with ALP in the final state in proton-proton collisions at the LHC. In particular, $j+\gamma+a, t+j+a$, and $t \bar{t}+a$ are studied considering a realistic detector simulation including the main background processes.

\section{A. ALP production with a photon and a jet}

We examine the potential of an ALP production associated with a photon and a jet to probe the parameter space of the model at the LHC. Although ALP is not directly detected by the LHC detectors, its production could be deduced from observation of events with an imbalance in the transverse momentum.

The production of an ALP associated with a photon and jet that has a final state of a photon, a jet, and missing transverse momentum is identifiable with large efficiency and purity. Figure 1 shows the representative Feynman diagrams for $j+\gamma+a$ in proton-proton collisions at the LHC. One of the interesting features of this process is its sensitivity to all couplings of ALP, i.e., $c_{W W}, c_{B B}, c_{G G}$, and $c_{a \Phi}$.

Assuming a single nonvanishing ALP coupling at a time, the leading order cross sections $\sigma(p p \rightarrow j+\gamma+a)\left(c_{X X}\right)$ read

$$
\begin{aligned}
& \sigma\left(c_{G G}\right)=30.0\left(\frac{c_{G G}}{f_{a}}\right)^{2} \mathrm{pb}, \quad \sigma\left(c_{W W}\right)=13.9\left(\frac{c_{W W}}{f_{a}}\right)^{2} \mathrm{pb}, \\
& \sigma\left(c_{B B}\right)=14.4\left(\frac{c_{B B}}{f_{a}}\right)^{2} \mathrm{pb}, \quad \sigma\left(c_{a \Phi}\right)=0.36\left(\frac{c_{a \Phi}}{f_{a}}\right)^{2} \mathrm{pb},
\end{aligned}
$$




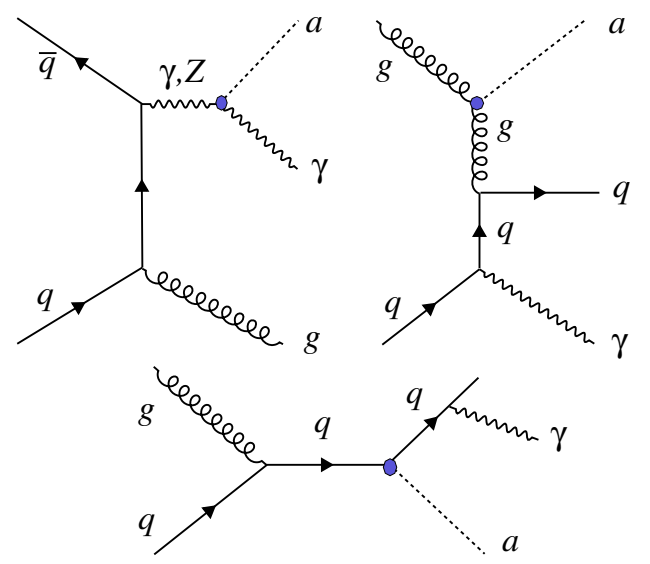

FIG. 1. Representative Feynman diagrams for production of an axionlike particle in association with a jet and a photon in protonproton collisions at the LHC.

where $f_{a}$ is in $\mathrm{TeV}$ unit. The cross sections are obtained with MadGraph5_aMC@NLO using the NNPDF23 [27] as the proton parton distribution function (PDF). The cross sections are calculated for the value of ALP mass $m_{a}=$ $1 \mathrm{MeV}$ and are found to be almost independent of $m_{a}$ for any value of $m_{a} \lesssim$ few $\mathrm{GeV}$ which is expected due to the fact that $m_{a}$ is negligible comparing to the typical energy scale of the $j+\gamma+a$ process. The dependence of the rate of $j+\gamma+a$ on the couplings of ALP to gluon and weak gauge bosons are shown in Fig. 2. For large values of $m_{a}$, the cross section decreases because of the low probability of production of heavy particle in final state. Among various processes in $j+\gamma+a$ production, those with a gluon and a quark in the initial state have larger contribution to the cross section than those from quark-antiquark annihilation. This is because of the large gluon PDF in particular at low $x$ where the process mostly occurs. As it can be seen in Eq. (8), there is more sensitivity to the ALP coupling to gluon, i.e., $c_{G G}$, which is due to the fact that it appears in processes with both initial states $g q(\bar{q})$ and $q \bar{q}$ as

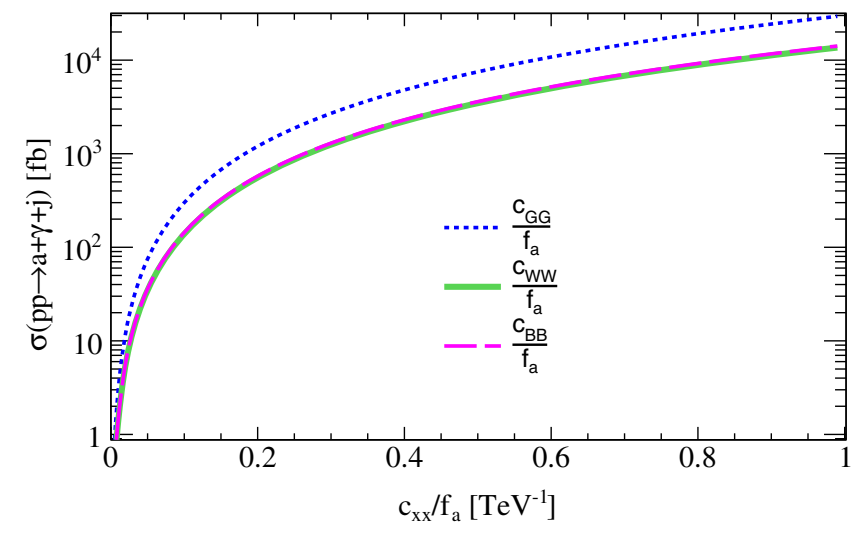

FIG. 2. Production cross section of $j+\gamma+a$ as a function of couplings $c_{G G}, c_{B B}, c_{W W}$ at the LHC with the center-of-mass energy of $14 \mathrm{TeV}$. well as the large gluon PDF in the $g q(\bar{q})$ part. As it is obvious, the cross section has a negligible dependence on $c_{a \Phi}$ coupling, $\sigma \sim \mathcal{O}\left(10^{-1}\right) \mathrm{fb}$. Therefore, in this work the effect of $\mathbf{O}_{a \Phi}^{\psi}$ operator in $j+\gamma+a$ channel is not studied. Using the beam dump experiments, the linear combination of $c_{W W}$ and $c_{B B}$ has been measured to be very small [11]

$$
\left|\frac{c_{B B}}{f_{a}} \cos \theta_{W}^{2}+\frac{c_{W W}}{f_{a}} \sin \theta_{W}^{2}\right| \leq 2.5 \times 10^{-3} \text { for } m_{a} \leq 1 \mathrm{MeV} .
$$

Therefore, in this work, we skip $c_{B B} / f_{a}$ coupling and we obtain the limit on $c_{B B} / f_{a}$ using the limit on $c_{W W} / f_{a}$.

The main background contributions to signal $(j+\gamma+a)$ arise from the following sources:

(i) Irreducible background from $Z \gamma+j$ events, where $Z$ boson decays to invisible neutrinos $Z \rightarrow \nu \bar{\nu}$.

(ii) $W \gamma+j, t \bar{t} \gamma$, and $t \gamma j$ processes with the $W$ boson (directly produced or from the top quark decay) decays into $l(=e, \mu, \tau)+\nu$, where $l$ is out of detector acceptance. In these processes, neutrino (s) from $W$ boson decays is a source of genuine missing transverse momentum.

(iii) $\gamma+j$ events where missing transverse momentum arises from leptonic decays of hadrons inside the jets, mis-measurement of jet energy, or detector noise. $W \gamma+j, t \bar{t} \gamma$, and $t \gamma j$ with hadronic decays of the $W$ boson and top quark(s) are also of this type.

The $Z \gamma+j$ and $W \gamma+j$ backgrounds are estimated based on a data-driven technique while the rest are calculated from simulation. Now, we turn to the simulation of the signal and SM background processes. The signal and background events are generated with MadGraph5_aMC@NLO and passed through Pythia [28] for showering, hadronization, and decays of unstable particles. Then, the events are passed to Delphes [29] for simulation of an upgraded CMS detector [30,31], including additional proton-proton interactions per bunch crossing (pileup) with an average of 200. The jet finding is performed via Fast Jet [32]. The anti- $k_{t}$ algorithm is used for jet reconstruction with a distance parameter of 0.4 [33] including pileup correction. To generate signal events, we consider the effect of one coupling at a time and various samples with ALP masses from 1 to $300 \mathrm{MeV}$ for $c_{G G}$ are generated and $f_{a}$ is set to $1 \mathrm{TeV}$. To find the exclusion regions in the parameter space $\left(c_{G G}, m_{a}\right)$, the signal selection efficiencies are obtained as a function of $c_{G G}$ and $m_{a}$. For simplicity, the search for the sensitivity on $c_{W W}$ coupling is performed only for one ALP mass benchmark of $m_{a}=1 \mathrm{MeV}$.

It should be noted that a fraction of ALPs decay inside the detector volume which would not appear as missing energy. The ALP decay length $L_{a}$ is proportional to $\sqrt{\gamma^{2}-1} / \Gamma_{a}$, where $\gamma$ and $\Gamma_{a}$ are the ALP Lorentz factor in each event and its total width, respectively. The probability that the ALP 
decays in the detector is proportional to $e^{-L_{\mathrm{det}} / L_{a}}$, where $L_{\mathrm{det}}$ is the distance from the collision point to the detector component in which the ALP is reconstructed. In this analysis, the probability that the ALP escapes the detector is considered event by event. As mentioned previously, the total width of the ALP, $\Gamma_{a}$, is taken from Ref. [23], where the width has been calculated using the chiral perturbation theory and vector meson dominance model.

Events are selected by requiring exactly one isolated photon with a transverse momentum $p_{\mathrm{T}} \geq 40 \mathrm{GeV}$ and a pseudorapidity $|\eta| \leq 3$.0. Photon isolation is applied using a pileup corrected isolation variable $I_{\text {rel }}$ as defined in Ref. [29] which assures negligible activity in the vicinity of the photon. $I_{\text {rel }}$ is obtained from the amount of transverse energy $p_{\mathrm{T}}$, calculated relatively to the photon $p_{\mathrm{T}}$, in a cone of radius $R=\sqrt{\eta^{2}+\phi^{2}}=0.3$ around the photon candidate, where $\phi$ is the azimuthal angle with respect to the $z$ direction. The selected photon is required to satisfy $I_{\text {rel }}<0.15$. Also, it is required to have at least one jet with $p_{\mathrm{T}} \geq 30 \mathrm{GeV}$ and $|\eta| \leq 4.0$. In order to suppress backgrounds with high jet multiplicity such as $t \bar{t} \gamma$, the number of jets is required to be less than three with at most one $\mathrm{b}$ jet. To have well-isolated objects, the angular separation between photon and jets, and between jets are required to be greater than 0.4 , i.e., $\Delta R(i, j)=$ $\sqrt{\left(\Delta \eta_{i j}\right)^{2}+\left(\Delta \phi_{i j}\right)^{2}} \geq 0.4$. The minimum cut on missing transverse energy $E_{T}^{\text {miss }}$ is chosen so that the analysis sensitivity to each ALP coupling is maximized. For the signal scenario of nonzero $c_{G G}$, the optimized cut on $E_{T}^{\text {miss }}$ is found to be $90 \mathrm{GeV}$ while for the $c_{W W}$ the best sensitivity is achievable with $E_{T}^{\text {miss }} \geq 50 \mathrm{GeV}$. In order to achieve better sensitivity and suppress the background contributions, it is required that $H_{T} \geq 200 \mathrm{GeV}$, where $H_{T}$ is defined as the scalar sum of $p_{T}$ of the objects in the final state. Further $\gamma+j$ background suppression could be obtained by applying an upper cut on $\Delta \phi(j, \gamma)$. Events of $\gamma+j$ are expected to be back-to-back distributed mostly around $\Delta \phi(j, \gamma) \sim \pi$, while this not the case for signal due to the presence of an ALP in the final. Therefore, an upper cut of 2.7 is applied on $\Delta \phi(j, \gamma)$. It is notable that the cut on $E_{T}^{\text {miss }}$ would be able to fulfill the high level trigger (HLT) condition; for example, see Table 1 of Ref. [34] which belongs to the LHC Run I and Run II. However, for the HLLHC, one could use cross triggers like requiring an energetic-isolated photon and $E_{T}^{\text {miss }}$ or an energetic-isolated photon, and $H_{T}$ which would be reliable HLT paths. The efficiency of such triggers needs to be studied by the experimental collaborations and is beyond the scope of this work.

In order to ensure the validity of the effective Lagrangian, its suppression scale $\left(f_{a}\right)$ is required to be far above the typical energy scale of the process. As a result, one must require that the energy scale of the process $\sqrt{\hat{s}}$ to be much less than $f_{a}$ in each event. In the processes under study in this work where ALP is in the final state appearing as missing energy, $\sqrt{\hat{s}}$ is not fully measurable. Therefore, to ensure the validity of the effective theory, $f_{a}$ is compared to $E_{T}^{\text {miss }}$. In each event, it is required that $E_{T}^{\text {miss }}<f_{a}$. The same approach is applied in the rest of the paper in other processes.

The production of a $Z$ boson in association with a photon and jets followed by invisible $Z$ boson decay is an irreducible background. We estimate this background using a data-driven method which relies on $Z\left(\rightarrow \mu^{+} \mu^{-}\right) \gamma+j$ events and then compare the results with simulation. In this technique, the pair of muons $\left(\mu^{+} \mu^{-}\right)$is interpreted as missing momentum in the $Z(\rightarrow \nu \bar{\nu}) \gamma+j$ process keeping selection criteria on the objects. The number of $Z(\rightarrow \nu \bar{\nu}) \gamma+j$ events is measured as the number of $Z\left(\rightarrow \mu^{+} \mu^{-}\right) \gamma+j$ events corrected for the acceptance cuts efficiency $(A)$, detector efficiencies $\epsilon$, and the branching fraction ratio

$$
\text { correction factor }=\frac{\operatorname{Br}(Z \rightarrow \nu \bar{\nu})}{\operatorname{Br}\left(Z \rightarrow \mu^{+} \mu^{-}\right)} \times A \times \epsilon,
$$

where $\operatorname{Br}(Z \rightarrow \nu \bar{\nu})$ and $\operatorname{Br}\left(Z \rightarrow \mu^{+} \mu^{-}\right)$are the branching fractions of invisible and leptonic decays of $Z$ boson.

Similar technique is used for estimation of the $W \gamma+j$ background, where $W$ boson decays leptonically. However, additional correction due to different topology needs to be included. The number of estimated backgrounds using the above method is consistent with the prediction from simulation within $1 \%$.

Figure 3 represents the distribution of $E_{T}^{\text {miss }}$ for signal with $c_{G G} / f_{a}=0.5 \mathrm{TeV}^{-1}, m_{a}=1 \mathrm{MeV}$, and various backgrounds with an integrated luminosity of $3000 \mathrm{fb}^{-1}$. Table I shows the efficiencies for the signal scenarios of $c_{G G} / f_{a}=0.5 \mathrm{TeV}^{-1}, c_{W W} / f_{a}=0.5 \mathrm{TeV}^{-1}$, and the main background processes after applying different cuts. The

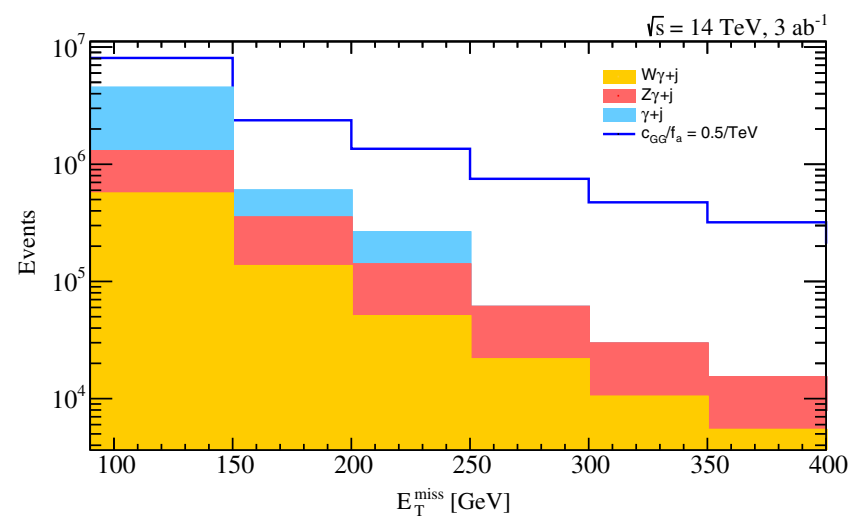

FIG. 3. Distribution of $E_{T}^{\text {miss }}$ for signal $\left(c_{G G} / f_{a}=0.5 \mathrm{TeV}^{-1}\right.$, $m_{a}=1 \mathrm{MeV}$ ) (blue line) $W \gamma+j$ (orange), $Z \gamma+j$ (red), and $\gamma+$ $j$ (blue) for an integrated luminosity of $3000 \mathrm{fb}^{-1}$ at the LHC with $\sqrt{s}=14 \mathrm{TeV}$. 
TABLE I. Efficiency for the signal and dominant SM backgrounds. The signal scenarios are corresponding to values of $c_{G G} / f_{a}=0.5 \mathrm{TeV}^{-1}, c_{W W} / f_{a}=0.5 \mathrm{TeV}^{-1}$, and $m_{a}=1 \mathrm{MeV}$.

\begin{tabular}{lccccc}
\hline \hline Cut & $c_{G G} / f_{a}=0.5 \mathrm{TeV}^{-1}$ & $c_{W W} / f_{a}=0.5 \mathrm{TeV}^{-1}$ & $Z \gamma+j$ & $W \gamma+j$ & $\gamma+j$ \\
\hline Jet and photon & 0.591 & 0.728 & 0.636 & 0.28 & 0.431 \\
$\Delta \phi(j, \gamma) \leq 2.7$ & 0.503 & 0.402 & 0.464 & 0.163 & 0.087 \\
$E_{T}^{\text {miss }} \geq 50 \mathrm{GeV}$ & 0.462 & $\mathbf{0 . 1 4 7}$ & 0.370 & 0.084 & $3.3 \times 10^{-4}$ \\
$E_{T}^{\text {miss }} \geq 90 \mathrm{GeV}$ & $\mathbf{0 . 3 6 5}$ & 0.031 & 0.216 & 0.033 & $3.7 \times 10^{-5}$ \\
\hline \hline
\end{tabular}

total number of background events corresponding to $3000 \mathrm{fb}^{-1}$ after $E_{T}^{\text {miss }} \geq 90 \mathrm{GeV}$ is $5.45 \times 10^{6}$.

Constraints on the ALP coupling $c_{G G} / f_{a}$ and mass derived from $j+\gamma+a$ channel with $3 \mathrm{ab}^{-1}$ integrated luminosity of data are presented as blue-dashed region in Fig. 4. The region denoted by supernova neutrino is obtained from supernova neutrino burst duration [35]. The regions labeled by $K^{ \pm} \rightarrow \pi^{ \pm}+a$ and "beam dump" denote the bounds from Kaon decay and from the proton beam dump experiment CHARM, respectively [35-37]. As can be seen, better sensitivity for lighter ALPs is obtained. The $95 \%$ confidence level (CL) expected upper limits on $c_{G G} / f_{a}$ and $c_{W W} / f_{a}$ for $m_{a}=1 \mathrm{MeV}$ with $3000 \mathrm{fb}^{-1}$ of data are found to be

$\left|c_{G G} / f_{a}\right| \leq 0.012 \mathrm{TeV}^{-1}, \quad\left|c_{W W} / f_{a}\right| \leq 0.041 \mathrm{TeV}^{-1}$.

The constraint on $c_{G G} / f_{a}$ at $95 \%$ CL from monojet events from the LHC experiments using $19.6 \mathrm{fb}^{-1}$ of $8 \mathrm{TeV}$ data is

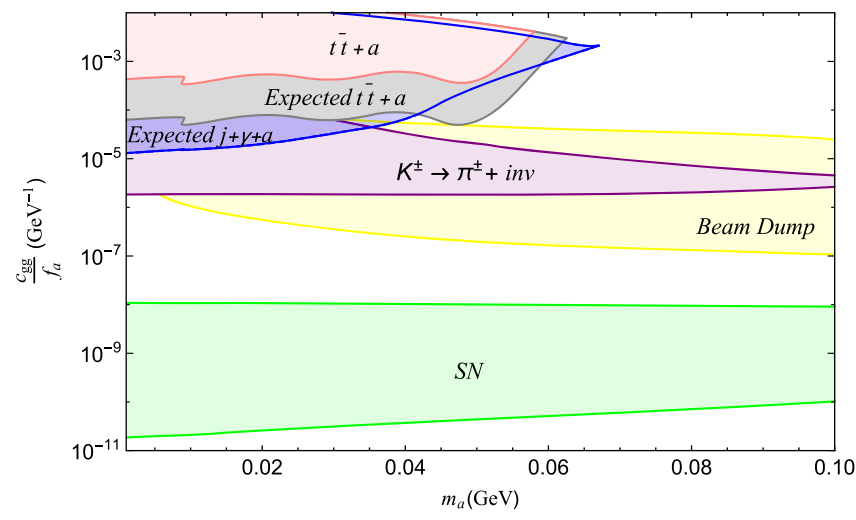

FIG. 4. The 95\% CL expected excluded regions of the parameter space of the ALP model $\left(c_{G G} / f_{a}, m_{a}\right)$ derived from $j+\gamma+a$ and $t \bar{t}+a$ channels. The regions assuming a new result of the collider search using the $t \bar{t}+a$ process are shown with integrated luminosities of 2.2 and $3000 \mathrm{fb}^{-1}$ at the center-of-mass energies of 13 and $14 \mathrm{TeV}$, respectively. The region denoted by SN shows the constraint from supernova neutrino burst duration taken from Ref. [35]. The regions labeled by $K^{ \pm} \rightarrow \pi^{ \pm}+a$ and beam dump present the constraints from Kaon decay and from the proton beam dump experiment CHARM taken from Ref. [35].
$0.025 \mathrm{TeV}^{-1}$ [13]. The $j+\gamma+a$ process provides comparable bound on $c_{G G}$ to the one from monojet events, and it could be a complementary channel to the monojet channel. The $95 \% \mathrm{CL}$ expected upper limits on $c_{W W}$ from the LHC at $\sqrt{s}=13 \mathrm{TeV}$ with $3000 \mathrm{fb}^{-1}$ from different channels are $[11,13]$ as follows:

$$
\begin{aligned}
& \left|c_{W W} / f_{a}\right| \leq 0.14 \mathrm{TeV}^{-1} \text { monophoton, } \\
& \left|c_{W W} / f_{a}\right| \leq 0.05 \mathrm{TeV}^{-1} \text { mono- } Z, \\
& \left|c_{W W} / f_{a}\right| \leq 0.16 \mathrm{TeV}^{-1} \text { mono- } W .
\end{aligned}
$$

The bound obtained from mono- $W$ channel is the weakest, and the strongest limit is from the mono- $Z$ process. One can see that the $j+\gamma+a$ process in this study provides better sensitivity to $c_{W W}$ with respect to the other processes.

\section{B. ALP production in association with a single top quark}

In this subsection, we discuss the potential of the production of an ALP in association with a top quark at the LHC. Single top quark can be produced in association with an ALP via three different mechanisms: t channel, $s$ channel, and tW production channel, where the t channel has the largest cross section. We concentrate on studying the single top quark production in association with an ALP in the $t$ and $s$ channels. Figure 5 shows representative Feynman diagrams for this process. The process is sensitive to $c_{W W}$ and has negligible sensitivity to $c_{a \Phi}$. The crosssection dependence on $c_{W W}$, assuming $m_{a}=1 \mathrm{MeV}$, has the following form:

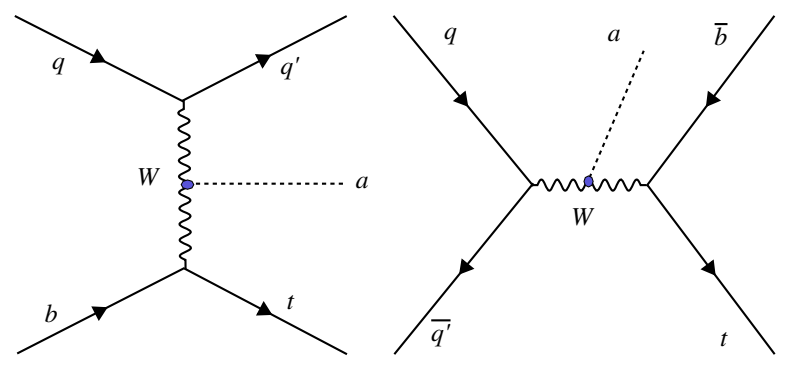

FIG. 5. Representative Feynman diagrams for production of an ALP with a top quark in the t- and s-channel modes at the LHC. 
TABLE II. Efficiency of signal with $c_{W W} / f_{a}=0.1,0.5 \mathrm{TeV}^{-1}, m_{a}=1 \mathrm{MeV}$, and of different background processes after applying all cuts are presented.

\begin{tabular}{lcccccc}
\hline \hline Signal $\left(c_{W W} / f_{a}=0.1(0.5) \mathrm{TeV}^{-1}\right)$ & $t+j$ & $t+W$ & $t \bar{t}$ & $W+j$ & $Z+j$ & $W Z$ \\
\hline $5.11 \%(5.16 \%)$ & $0.014 \%$ & $0.02 \%$ & $0.01 \%$ & $0.02 \%$ & $0.0001 \%$ & $0.08 \%$ \\
\hline \hline
\end{tabular}

$$
\sigma(p p \rightarrow t+j+a)=0.12 \times\left(\frac{c_{W W}}{f_{a}}\right)^{2} \mathrm{pb}
$$

The search uses events with a top quark, at least one light-flavor jet, and missing transverse energy with the top quark decays into a bottom quark and a $W$ boson, followed by the $W$ decay into a charged lepton $(e, \mu)$ and a neutrino. The final state consists of an electron or a muon, missing transverse energy, and at least two jets from which one (or both) should originate from the hadronization of a b quark.

The main backgrounds processes to the signal are $t \bar{t}, \mathrm{SM}$ single top processes (t, s, and tW channels), $W+j, Z+j$, and $W Z$ productions. The event generation, detector simulation, and reconstruction are performed with similar tools and techniques as in Sec. III A. To select signal events, we are required to have one isolated charged lepton $(e, \mu)$ with $p_{\mathrm{T}} \geq 20 \mathrm{GeV}$ and $|\eta| \leq 3.0$. In addition, each event is required to have two or three jets with $p_{\mathrm{T}} \geq 20 \mathrm{GeV}$, out of which at least one should be tagged as a b jet with $|\eta| \leq 3.5$. To ensure all selected objects are well isolated, the angular separation between all objects should be $\Delta R \geq 0.4$. The optimized lower cut on $E_{T}^{\text {miss }}$ is $250 \mathrm{GeV}$ which reduces the contribution of all SM background processes significantly. All the efficiency for the signal and SM backgrounds after final cuts are represented in Table II. The total number of background events after all cuts with an integrated luminosity of $3000 \mathrm{fb}^{-1}$ is $1.41 \times 10^{6}$.

The expected upper limit on $c_{W W} / f_{a}$ at $95 \% \mathrm{CL}$ for $m_{a}=1 \mathrm{MeV}$ is obtained as

$$
\left|c_{W W} / f_{a}\right| \leq 0.75 \mathrm{TeV}^{-1}
$$

The limit is looser than the those obtained from $j+\gamma+a$ in Sec. III A. This was expected as the dependence of $t+j+a$ cross section to $c_{W W} / f_{a}$ is weaker than the cross section of $j+\gamma+a$ by 2 orders of magnitude.

\section{ALP production in association with a pair of $t \bar{t}$}

In this part, we investigate the potential of the top quark pair production in association with an ALP at the LHC. Representative leading order Feynman diagrams for $t \bar{t}+a$ production are depicted in Fig. 6. The process has a considerable sensitivity to $c_{G G}$ and has low sensitivity to $c_{a \Phi}$. The inclusive cross section up to order $f_{a}^{-2}$ for $m_{a}=1 \mathrm{MeV}$ can be written as

$$
\begin{aligned}
& \sigma(p p \rightarrow t \bar{t}+a)=3.9 \times\left(\frac{c_{G G}}{f_{a}}\right)^{2} \mathrm{pb} \\
& \sigma(p p \rightarrow t \bar{t}+a)=0.04 \times\left(\frac{c_{a \Phi}}{f_{a}}\right)^{2} \mathrm{pb} .
\end{aligned}
$$

At the LHC, the $t \bar{t}+a$ process can be probed directly via the $t \bar{t}+E_{T}^{\text {miss }}$ signature. In Refs. [38,39], the CMS and ATLAS Collaborations performed searches for simplified models for dark matter production assuming the existence of a mediator that couples to both the dark matter and SM particles. Particularly, the concentration of these searches is on the case of production of a fermionic dark matter via the exchange of a color-neutral scalar or pseudoscalar particle. In these models, the coupling between the new (pseudo) scalar state and SM particles is Yukawa-like as a result proportional to the fermion masses. It follows that the scalar mediator would be produced mostly in association with heavy quarks (in particular top quark) or via loop induced gluon fusion. The characteristic signature to search for dark matter in $t \bar{t}+\phi(\rightarrow \chi \chi)$ channel is the presence of a high missing transverse momentum recoiling against $t \bar{t}$. This signature is similar to $t \bar{t}+a$. Therefore, we reinterpret the results of the CMS experiment search at the center-of-mass energy $13 \mathrm{TeV}$ to the ALP model and extrapolate the results to larger integrated luminosity of data at the center-of-mass energy of $14 \mathrm{TeV}$.

We focus on the semileptonic $t \bar{t}$ channel and follow similar selection as applied on the CMS experiment search [38]. Events are selected by requiring one isolated lepton $(e, \mu)$ with $p_{\mathrm{T}} \geq 30 \mathrm{GeV}$ and $|\eta|<2.5$, at least three jets

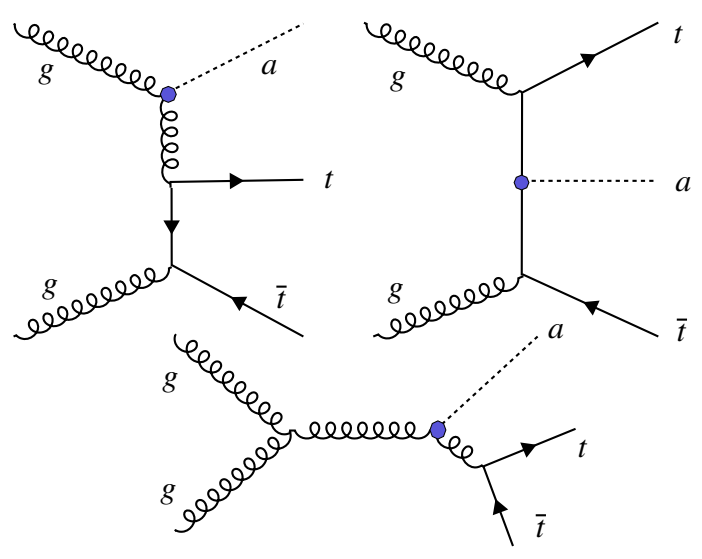

FIG. 6. Leading order Feynman diagrams describing the production of a pair of top quark with an ALP at the LHC. 
from which one has to be a b-tagged jet. Jets are required to have $p_{\mathrm{T}} \geq 30 \mathrm{GeV}$ and $|\eta| \leq 2.4$. Events with additional charged leptons with $p_{\mathrm{T}} \geq 10 \mathrm{GeV}$ that satisfy loose identification criteria are rejected. The magnitude of missing transverse momentum should be greater than $160 \mathrm{GeV}$. For reducing the background contribution from $t \bar{t}$ and $W+j$, the transverse mass $M_{\mathrm{T}}=$ $\sqrt{2 p_{T, l} E_{T}^{\text {miss }}\left(1-\cos \Delta \phi\left(\vec{p}_{T, l}, \vec{E}_{T}^{\text {miss }}\right)\right)}$ is required to be larger than $160 \mathrm{GeV}$. The magnitude of the vector sum of jets with $p_{T} \geq 20 \mathrm{GeV}$ and $|\eta| \leq 5.0, \mathrm{H}_{\mathrm{T}}^{\text {miss }}$, is required to be greater than $120 \mathrm{GeV}$. For further suppression of SM $t \bar{t}$, additional cut on $\mathrm{M}_{\mathrm{T} 2}^{\mathrm{W}}$, introduced in Ref. [40], is applied. It is required that $\mathrm{M}_{\mathrm{T} 2}^{\mathrm{W}} \geq 200 \mathrm{GeV}$. The predicted number of background events corresponding to $2.2 \mathrm{fb}^{-1}$ is 43.2. The corresponding expected upper bounds on $c_{G G} / f_{a}$ with the integrated luminosities of 2.2 and $100 \mathrm{fb}^{-1}$ for $m_{a}=1 \mathrm{MeV}$ are found to be

$$
\begin{aligned}
& \frac{c_{G G}}{f_{a}} \leq 0.44 \mathrm{TeV}^{-1} @ 2.2 \mathrm{fb}^{-1}, \\
& \frac{c_{G G}}{f_{a}} \leq 0.15 \mathrm{TeV}^{-1} @ 100 \mathrm{fb}^{-1} .
\end{aligned}
$$

Assuming the same selection as those used in $13 \mathrm{TeV}$ analysis, prospects at $14 \mathrm{TeV}$ at HL-LHC is obtained. The constraints for $m_{a}=1 \mathrm{MeV}$ at the center-of-mass energy of $14 \mathrm{TeV}$ at 300 and $3000 \mathrm{fb}^{-1}$ are found to be

$$
\begin{aligned}
& \frac{c_{G G}}{f_{a}} \leq 0.11 \mathrm{TeV}^{-1} @ 300 \mathrm{fb}^{-1}, \\
& \frac{c_{G G}}{f_{a}} \leq 0.063 \mathrm{TeV}^{-1} @ 3000 \mathrm{fb}^{-1} .
\end{aligned}
$$

Regions in the ALP coupling $c_{G G}$ and mass at $95 \% \mathrm{CL}$, from $t \bar{t}+a$ process are presented in Fig. 4. The regions are corresponding to integrated luminosities of 2.2 and $3000 \mathrm{fb}^{-1}$. As can be seen in Fig. 4, the result obtained

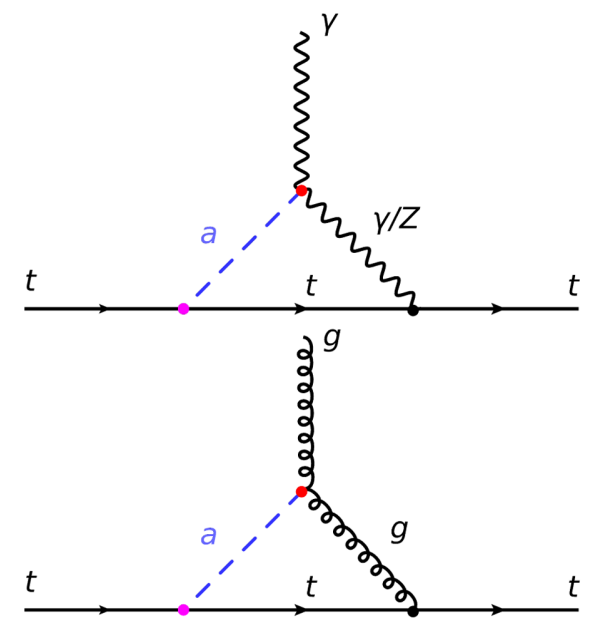

from this channel is complementary to that derived from $j+\gamma+a$ process. For the region $m_{a} \lesssim 0.04 \mathrm{GeV}$, the $j+\gamma+a$ process shows better sensitivity to $c_{G G} / f_{a}$, while in the mass region above $\sim 0.04 \mathrm{GeV}, t \bar{t}+a$ channel provides stronger bounds.

\section{CONSTRAINING THE ALP MODEL BY THE TOP QUARK DIPOLE MOMENTS}

Several types of experiments are used to search for the ALP model, ranging from the searches for direct production of ALP at colliders to those from cosmological and astroparticle physics experiments. However, a complementary approach in hunting for ALP effects would be the examination of the ALP indirect effects in higher order processes. In this section, we derive sensitivity on the ALP coupling using the upper bounds on the top quark (chromo) magnetic dipole moment ((C)MDM). Top quark dipole moments are useful observables which enable us to constrain the parameter space of new physics models $[41,42]$. It is worth mentioning here that there are also studies to explain the anomalous magnetic moment of the muon by $\left((g-2)_{\mu} / 2\right)$ employing a light pseudoscalar boson or ALP $[7,14,43]$.

The ALP interactions with photon, $Z$ boson, gluons, and top quark as described in Lagrangian presented in Eq. (3) lead to considerable contributions to the top quark dipole moments. The contributions from the ALP model to the top quark MDM are shown in the top plots demonstrated in Fig. 7 and those contributing to the top quark CMDM are shown in the bottom plots in Fig. 7.

It is notable that the dipole moments are proportional to two of the couplings of ALP at a time, $c_{a \Phi}$ and $c_{G G}$ for chromodipole moments or $c_{W W(B B)}$ for electroweak dipole moments. Therefore, dipole moments provide the possibility of probing two ALP couplings at a time. The ALP model contributes to the top quark MDM $\left(a_{t}\right)$ and CMDM $\left(\tilde{a}_{t}\right)$ according to the following relations:

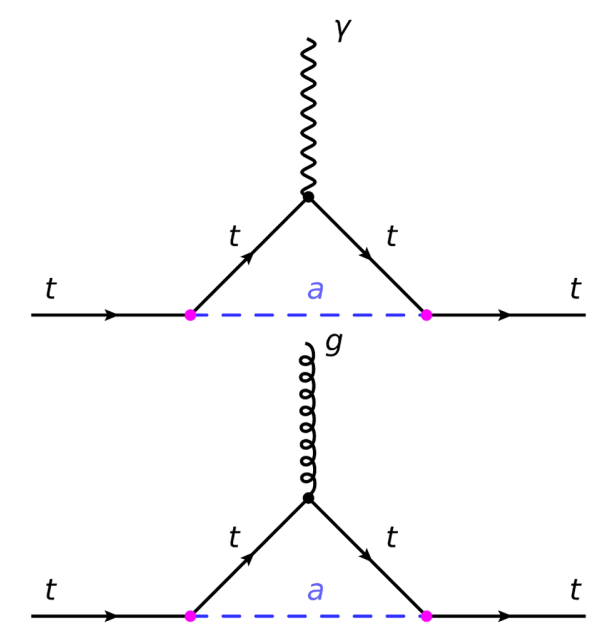

FIG. 7. Feynman diagrams with contributions from an ALP to the top (chromo)electric and (chromo)magnetic dipole moments. 

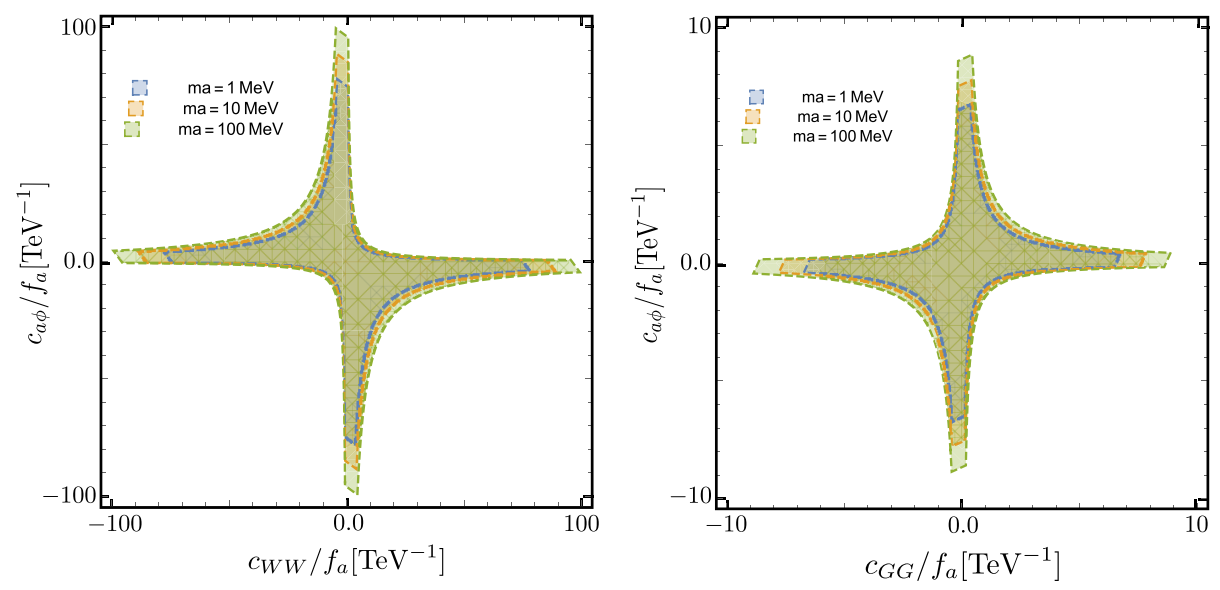

FIG. 8. The $95 \%$ CL exclusion limits on $\left(c_{a \Phi} / f_{a}, c_{W W} / f_{a}\right)$ (left) and $\left(c_{a \Phi} / f_{a}, c_{G G} / f_{a}\right)$ (right) using the top quark MDM and CMDM limits.

$a_{t}=\frac{g_{t}-2}{2}=\frac{c_{a \Phi} \times c_{W W}}{f_{a}^{2}} \frac{m_{t}^{2} \sin ^{2} \theta_{w}}{\pi^{2}}\left(\frac{3}{4}+\log \left(\frac{\Lambda}{m_{a}}\right)\right)$,

$\tilde{a}_{t}=\frac{c_{a \Phi} \times c_{G G}}{f_{a}^{2}} \frac{m_{t}^{2}}{\pi^{2}}\left(\frac{3}{4}+\log \left(\frac{\Lambda}{m_{a}}\right)\right)$.

As it can be seen, there is a logarithmic divergence to the dipole moments from the ALP model in photon (gluon) loops. The contribution of the loop with $Z$ boson exchange is proportional to $\log \left(\Lambda^{2} / m_{Z}^{2}\right)$ which is suppressed by the $Z$ boson mass therefore neglected here. The factor $\Lambda$ in the numerator of the logarithms which comes from the loop divergences could be naturally assumed to be equal to the new physics scale. In the ALP model, $\Lambda$ could be taken equal to $f_{a}$. Using the upper limits on the top quark MDM and CMDM, one is able to constrain the ALP model parameters.

There are several studies to constrain the top quark dipole moments using the collider experiments and indirect searches which could be found in Refs. [44-51]. Limits on the top MDM obtained as $-3 \leq a_{t} \leq 0.45$ from the $t \bar{t} \gamma$ production at the Tevatron and the LHC. The CMS experiment limits at $95 \% \mathrm{CL}$ on the CMDM of the top quark is $-0.043 \leq \tilde{a}_{t} \leq 0.117$ [46].

Figure 8 shows the 95\% CL exclusion regions on $\left(c_{a \Phi} / f_{a}, c_{W W} / f_{a}\right)$ (left panel) and $\left(c_{a \Phi} / f_{a}, c_{G G} / f_{a}\right)$ (right panel) obtained from the top quark MDM $\left(a_{t}\right)$ and CMDM $\left(\tilde{a}_{t}\right)$ limits, respectively. The bounds are depicted for three values for the mass ALP $m_{a}=1,10,100 \mathrm{MeV}$. For smaller value of ALP mass, tighter bounds are achieved.

The constraints are complementary to those derived from collider searches. In particular, for very light ALP masses, the magnetic dipole moments are powerful tools for exploration of the model parameters.

\section{SUMMARY AND CONCLUSIONS}

Many SM extensions commonly predict the existence of light $C P$-odd scalars, so called axionlike particles, which can couple to the SM gauge bosons and matter fields. Such scalars arise from spontaneously broken global symmetries and provide hints to answer some theoretical and observational defects of the SM such as dark matter, baryogenesis, and strong $C P$ problem.

In this study, to search for the new physics effects from ALPs, we consider the most general effective Lagrangian up to dimension five which describes the ALP interactions with SM fields. We present collider limits on ALP model parameters from the $j+\gamma+a, t+j+a$, and $t \bar{t}+a$ search channels at the LHC with an integrated luminosity of $3000 \mathrm{fb}^{-1}$ of data. It is found that the $j+\gamma+a$ channel is a promising process to probe both $c_{W W}$ and $c_{G G}$. In particular, the coupling of ALP with photon is reachable using this channel down to $\left|c_{W W} / f_{a}\right| \leq 0.041 \mathrm{TeV}^{-1}$ for

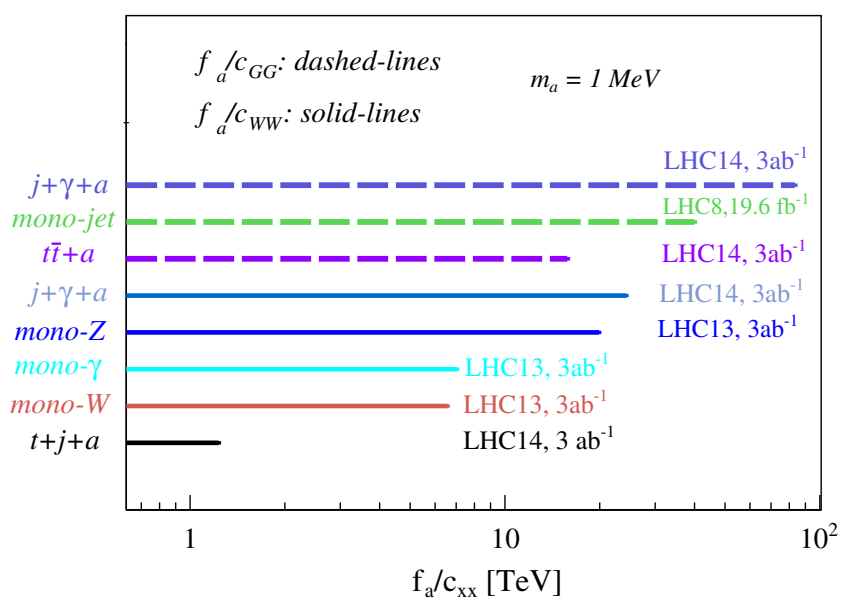

FIG. 9. The prospects for limits on $f_{a} / c_{G G}$ and $f_{a} / c_{W W}$ in TeV unit for $m_{a}=1 \mathrm{MeV}$ at HL-LHC are presented. The results shown as $t+j+a$, top pair $+a$, and $j+\gamma+a$ are derived from the current study. The other limits are taken from Refs. [11,13]. All limits are corresponding to an integrated luminosity of $3000 \mathrm{fb}^{-1}$ at $\sqrt{s}=14 \mathrm{TeV}$ except the one from mono-jet channel which obtained from $8 \mathrm{TeV}$ LHC collisions data. 
$m_{a}=1 \mathrm{MeV}$, which is at the order of the best expected limit from mono- $Z$ process. Reasonable sensitivity to the coupling of ALP to gluons $c_{G G} / f_{a} \leq 0.012 \mathrm{TeV}^{-1}$ and $c_{G G} / f_{a} \leq 0.063 \mathrm{TeV}^{-1}$ for $m_{a}=1 \mathrm{MeV}$ are obtained from the $j+\gamma+a$ and $t \bar{t}+a$ processes, respectively. Regions at the $95 \%$ CL in the ALP parameter space $\left(c_{G G}, m_{a}\right)$ are found using $j+\gamma+a$ and $t \bar{t}+a$ channels which shows that the channels are complementary in probing the ALP coupling to gluon.

In Fig. 9, our prospect for bounds on the new physics energy scale from the ALP model over the couplings $f_{a} / c_{G G}$ and $f_{a} / c_{W W}$ in $\mathrm{TeV}$ unit with $m_{a}=1 \mathrm{MeV}$ are presented. The bounds are the $95 \% \mathrm{CL}$ and correspond to $3000 \mathrm{fb}^{-1}$ of LHC data at the center-of-mass energies of 13 and $14 \mathrm{TeV}$. The prospects for bounds from various channels are shown as well which are taken from Refs. $[11,13]$. All limits are corresponding to an integrated luminosity of $3000 \mathrm{fb}^{-1}$ at $\sqrt{s}=14 \mathrm{TeV}$ except for the one obtained from the monojet process which has been obtained from the LHC monojet analysis at $8 \mathrm{TeV}$ collisions with $19.6 \mathrm{fb}^{-1}$ of integrated luminosity of data.

At the end, we show that the ALPs could have a significant contribution to the top quark (chromo)magnetic dipole moment. Using the present limits on the top quark MDM and CMDM, the ALP couplings $c_{a \Phi} / f_{a}, c_{W W} / f_{a}$, and $c_{a \Phi} / f_{a}, c_{G G} / f_{a}$ are constrained simultaneously. The exclusions regions are found to be tighter for lighter ALPs.

\section{ACKNOWLEDGMENTS}

We thank Andrea Giammanco for proposing data-driven ideas for background estimation, reading the manuscript, and giving several useful comments. The authors are grateful to Yotam Soreq for the insightful comments and for providing data of Ref. [23]. M. M. N. would like to thank Iran National Science Foundation for the support.
[1] G. 't Hooft, Phys. Rev. Lett. 37, 8 (1976).

[2] M. Dine, arXiv:hep-ph/0011376.

[3] A. Hook, arXiv:1812.02669.

[4] R. D. Peccei and H. R. Quinn, Phys. Rev. Lett. 38, 1440 (1977).

[5] M. J. Dolan, F. Kahlhoefer, C. McCabe, and K. SchmidtHoberg, J. High Energy Phys. 03 (2015) 171; 07 (2015) 103(E).

[6] C. Patrignani et al. (Particle Data Group), Chin. Phys. C 40, 100001 (2016).

[7] D. Chang, W. F. Chang, C. H. Chou, and W. Y. Keung, Phys. Rev. D 63, 091301 (2001).

[8] K. S. Jeong, T. H. Jung, and C. S. Shin, arXiv:1811.03294.

[9] B. Bellazzini, A. Mariotti, D. Redigolo, F. Sala, and J. Serra, Phys. Rev. Lett. 119, 141804 (2017).

[10] E. Arganda, A. D. Medina, N. I. Mileo, R. A. Morales, and A. Szynkman, Phys. Lett. B 789, 575 (2019).

[11] I. Brivio, M. B. Gavela, L. Merlo, K. Mimasu, J. M. No, R. del Rey, and V. Sanz, Eur. Phys. J. C 77, 572 (2017).

[12] J. Jaeckel and M. Spannowsky, Phys. Lett. B 753, 482 (2016).

[13] K. Mimasu and V. Sanz, J. High Energy Phys. 06 (2015) 173.

[14] M. Bauer, M. Neubert, and A. Thamm, Phys. Rev. Lett. 119, 031802 (2017).

[15] M. Bauer, M. Neubert, and A. Thamm, J. High Energy Phys. 12 (2017) 044.

[16] A. Mariotti, D. Redigolo, F. Sala, and K. Tobioka, Phys. Lett. B 783, 13 (2018).

[17] S. Knapen, T. Lin, H. K. Lou, and T. Melia, Phys. Rev. Lett. 118, 171801 (2017).

[18] A. M. Sirunyan et al. (CMS Collaboration), arXiv: 1810.04602 .

[19] R. Bruce et al., arXiv:1812.07688.
[20] C. Baldenegro, S. Fichet, G. von Gersdorff, and C. Royon, J. High Energy Phys. 06 (2018) 131.

[21] X. Cid Vidal, A. Mariotti, D. Redigolo, F. Sala, and K. Tobioka, J. High Energy Phys. 01 (2019) 113.

[22] M. B. Gavela, R. Houtz, P. Quilez, R. del Rey, and O. Sumensari, Eur. Phys. J. C 79, 369 (2019).

[23] D. Aloni, Y. Soreq, and M. Williams, arXiv:1811.03474.

[24] A. Alloul, N. D. Christensen, C. Degrande, C. Duhr, and B. Fuks, Comput. Phys. Commun. 185, 2250 (2014).

[25] C. Degrande, C. Duhr, B. Fuks, D. Grellscheid, O. Mattelaer, and T. Reiter, Comput. Phys. Commun. 183, 1201 (2012).

[26] J. Alwall, M. Herquet, F. Maltoni, O. Mattelaer, and T. Stelzer, J. High Energy Phys. 06 (2011) 128.

[27] R. D. Ball et al., Nucl. Phys. B867, 244 (2013).

[28] T. Sjostrand, S. Mrenna, and P. Z. Skands, J. High Energy Phys. 05 (2006) 026.

[29] J. de Favereau, C. Delaere, P. Demin, A. Giammanco, V. Lemaître, A. Mertens, and M. Selvaggi (DELPHES 3 Collaboration), J. High Energy Phys. 02 (2014) 057.

[30] S. Chatrchyan et al. (CMS Collaboration), J. Instrum. 3, S08004 (2008).

[31] D. Contardo, M. Klute, J. Mans, L. Silvestris, and J. Butler, Reports No. CERN-LHCC-2015-010, No. LHCC-P-008, and CMS-TDR-15-02.

[32] M. Cacciari, G. P. Salam, and G. Soyez, Eur. Phys. J. C 72, 1896 (2012).

[33] M. Cacciari, G. P. Salam, and G. Soyez, J. High Energy Phys. 04 (2008) 063.

[34] M. Aaboud et al. (ATLAS Collaboration), Eur. Phys. J. C 77, 317 (2017).

[35] H. Fukuda, K. Harigaya, M. Ibe, and T. T. Yanagida, Phys. Rev. D 92, 015021 (2015).

[36] E. Izaguirre, T. Lin, and B. Shuve, Phys. Rev. Lett. 118, 111802 (2017). 
[37] B. Dbrich, J. Jaeckel, F. Kahlhoefer, A. Ringwald, and K. Schmidt-Hoberg, J. High Energy Phys. 02 (2016) 018; 02 (2016) 018.

[38] A. M. Sirunyan et al. (CMS Collaboration), Eur. Phys. J. C 77, 845 (2017).

[39] M. Aaboud et al. (ATLAS Collaboration), Eur. Phys. J. C 78, 18 (2018).

[40] Y. Bai, H. C. Cheng, J. Gallicchio, and J. Gu, J. High Energy Phys. 07 (2012) 110.

[41] S. Khatibi and M. Mohammadi Najafabadi, Phys. Rev. D 90, 074014 (2014).

[42] S. Yaser Ayazi, S. Khatibi, and M. Mohammadi Najafabadi, J. High Energy Phys. 10 (2012) 103.

[43] W. J. Marciano, A. Masiero, P. Paradisi, and M. Passera, Phys. Rev. D 94, 115033 (2016).
[44] A. O. Bouzas and F. Larios, Phys. Rev. D 87, 074015 (2013).

[45] M. Fael and T. Gehrmann, Phys. Rev. D 88, 033003 (2013).

[46] CMS Collaboration, Report No. CMS-PAS-TOP-14-005.

[47] J. F. Kamenik, M. Papucci, and A. Weiler, Phys. Rev. D 85, 071501 (2012); 88, 039903(E) (2013).

[48] S. M. Etesami, S. Khatibi, and M. Mohammadi Najafabadi, Phys. Rev. D 97, 075023 (2018).

[49] J. A. Aguilar-Saavedra, B. Fuks, and M. L. Mangano, Phys. Rev. D 91, 094021 (2015).

[50] S. M. Etesami and E. D. Roknabad, arXiv:1810.07477.

[51] S. M. Etesami, S. Khatibi, and M. Mohammadi Najafabadi, Eur. Phys. J. C 76, 533 (2016). 